were performed on aged-matched Harlan mice (C57BL/6) using a previously established model of hypercholanaemic pregnancy. Serum biochemical measurements, global DNA methylation studies and qPCR was performed on mothers and offspring at a defined stage of pregnancy (day 18) and post-natal life in offspring (week 18). Statistical analysis for multiple comparisons was performed by repeated measures of ANOVA and Newman-Keuls post-hoc testing $(\mathrm{p}<0.05)$.

Results We analysed the lipid profile of fetal serum from untreated ICP, UDCA-treated ICP and uncomplicated pregnancies and found that UDCA ameliorates ICP-associated fetal dyslipidemia. We then investigated the effects of UDCA in a mouse model of hypercholanemic pregnancy and showed that it induces hepatoprotective mechanisms in the fetal liver, reduces hepatic fatty acid synthase (Fas) expression and improves glucose tolerance in the adult offspring. Finally, we show that ICP leads to epigenetic changes in pathways of relevance to the offspring phenotype.

Conclusion UDCA prevents adverse metabolic features in offspring exposed to hypercholanemia in pregnancy. In addition, the effects of ICP on the offspring appear to be mediated by epigenetic mechanisms.

\section{G349(P) EVALUATION OF DISEASE ACTIVITY BIOMARKERS IN AN INTERNATIONAL COHORT OF CHILDREN WITH LUPUS NEPHRITIS}

${ }^{1}$ Chiara De Mutiis, ${ }^{2}$ Kjell Tullus, International Lupus Nephritis Study Group in Children. ${ }^{1}$ Department of Paediatrics, Maggiore Hospital, Bologna, Italy; ${ }^{2}$ Department of Paediatric Nephrology, Great Ormond Street Hospital for Children NHS Foundation Trust, London, UK

\subsection{6/archdischild-2020-rcpch.298}

Introduction Systemic lupus erythematosus is a chronic and severe disease characterized by flares and remissions where renal involvement is strongly related to the long term prognosis. Close monitoring of the children with lupus nephritis is fundamental for long term survival. The aim of this study was to describe trends of routine laboratory biomarkers after initial treatment in an international cohort of children with SLE nephritis.

Methods Children ( $<17$ years old) with $\geq$ class III biopsy (ISN/RPS) diagnosed and treated in the last 10 years in 23 international centers were included.

Laboratory biomarkers at specific time points were retrospectively analyzed from diagnosis to 2 years follow-up and at last available follow-up and reported as medians (IQR).

Results 382 patients (278 girls), mean age 11 years, were included. $30 \%$ of patients were Asian, 23\% Indian, $18.5 \%$ Caucasian, 7.8\% Hispanic, 4\% Subs African/African American, 2\% Heterogeneous, $0.3 \%$ Semitic-Arabic.

At diagnosis eGFR was $94(64-119) \mathrm{ml} / \mathrm{min} / 1.73 \mathrm{~m}^{2}$ and increased to $105(83-128) \mathrm{ml} / \mathrm{min} / 1.73 \mathrm{~m}^{2}$ within the first month. Initially Urine-Protein/Urine-Creatinine (UPr:UC) was in nephrotic range in $48 \%$ of cases, $2(0.6-43) \mathrm{mg} / \mathrm{mg}$, and improved during the first 6 months but was still raised in $24 \%$ of patients at 12 month-follow-up. Serum albumin was $31(23-37) \mathrm{g} / \mathrm{L}$ and normalized within the first month in most children.

Haemoglobin was initially 99 (84.2-115) g/L and improved in 2 months, however about $40 \%$ of patients had persistent low values below $120 \mathrm{~g} / \mathrm{L}$ during follow-up. ESR was 49 (21-
76) $\mathrm{mm} / \mathrm{hr}$ and declined mainly during the first 3 months, but $49.7 \%$ of patients still had high ESR at 24 months.

At diagnosis double stranded DNA was raised to 194.5 (29-401) IU/ml, rapidly decreased in the first three months and only normalized in half of the patients. C3 was 0.38 $(0.8-0.6) \mathrm{g} / \mathrm{L}$ and $\mathrm{C} 4 \quad 0.07 \quad(0.04-0.15) \mathrm{g} / \mathrm{L}$ and improved within 2 months.

Conclusion In this cohort of children with lupus nephritis haemoglobin, serum albumin and complement showed the fastest improvements after start of treatment; often within two months. Other parameters like ESR, dsDNA and proteinuria took much longer to improve and in a substantial proportion of the children never reached normal values.

\section{G350(P) BILIARY ATRESIA: REFERRAL BARRIERS WITHIN THE COMMUNITY}

${ }^{1}$ EC Alexander, ${ }^{1} \mathrm{C}$ Burford, ${ }^{1} \mathrm{D}$ Thaxter, ${ }^{2} \mathrm{M}$ Davenport, ${ }^{1} \mathrm{~A}$ Dhawan, ${ }^{1} \mathrm{~V}$ Jain. ${ }^{1}$ Paediatric Liver, GI and Nutrition Centre and Mowatlabs, King's College Hospital, London, UK; ${ }^{2}$ Department of Paediatric Surgery, King's College Hospital, London, UK

\subsection{6/archdischild-2020-rcpch.299}

Aims Biliary atresia (BA) is an idiopathic neonatal cholangiopathy, requiring Kasai Portoenterostomy (KP), to re-establish bile drainage. Failure of KP necessitates liver transplantation (LT). Earlier age at KP $(<46$ days $)$ is associated with improved post-KP outcomes. Late referrals from the community (>100 days) often undergo primary LT. National guidelines state all term babies jaundiced after 14 days of age, to be referred for a conjugated bilirubin. The aim of this study was to identify referral pathways and barriers within the community for potential BA infants.

Methods A retrospective review of clinical notes, of 47 consecutive newly-diagnosed BA infants (Jan 2016-May 2018) at a specialised liver unit. Values expressed as median (range).

Results Forty-seven infants (22F:25M, 91\% isolated BA and $9 \%$ syndromic BA), were included. Age of referral from community to liver unit was 30 days (5-238 days); $83 \%$ were referred before 46 days; 2 patients referred $>100$ days. Seventy-seven percent $(36 / 47)$ of infant parents expressed concerns of jaundice at the 14-day community check, 19\% (9/47) had jaundice recognised appropriately by secondary care whilst inpatient for other co-morbidities, and 2/47 infants were referred early (5 and 6 days) following antenatal ultrasound abnormalities. Seventy-five percent (27/ 36) of the concerned parents were falsely reassured by health professionals. Two thirds of these infants were breast/ mixed-fed and 30\% were dark-skinned. These 27 infants ended up being referred to the liver unit at 43 days (17238 days), either by (i) persistent parental pressure (13) (ii) parental self-referral to A+E for jaundice (2) (iii) 6-8 week GP check (9) (iv) secondary care presentation for other comorbidities (3). Only 4/47 parents reported recognising stool was pale. No parent reported being shown a stool colour chart. Referral from community to liver unit admission was 3 days (0-28 days), and liver unit admission to KP was 8 days (2-48 days).

Conclusion Tighter regulation of the national jaundice guideline is required, to expedite referrals for potential BA from community to liver units, in turn, improving outcomes in this high-risk cohort of patients. 